\title{
ENHANCEMENT OF THE STABILITY OF LATTICE BOLTZMANN METHODS BY DISSIPATION CONTROL
}

\author{
ALEXANDER N. GORBAN ${ }^{\dagger}$ AND DAVID J. PACKWOOD ${ }^{\dagger}$
}

\begin{abstract}
Artificial dissipation is a well known tool for the improvement of stability of numerical algorithms. At the same time, this affects the accuracy of the computation. We analyze various approaches proposed for enhancement of the Lattice Boltzmann Methods (LBM) stability. In addition to the previously known methods, the Multiple Relaxation Time (MRT) models, the entropic lattice Boltzmann method (ELBM), and filtering (including entropic median filtering), we develop and analyze new filtering techniques with independent filtering of different modes.

All these methods affect the dissipation in the system and may adversely affect the reproduction of the proper physics. To analyze the effect of dissipation on accuracy and to prepare practical recommendations, we test the enhanced LBM methods on the standard benchmark, the 2D lid driven cavity on a coarse grid $(101 \times 101$ nodes $)$. The accuracy was estimated by the position of the first Hopf bifurcation points in these systems.

We find that two techniques, MRT and median filtering, succeed in yielding a reasonable value of the Reynolds number for the first bifurcation point. The newly created limiters, which filter the modes independently, also pick a reasonable value of the Reynolds number for the first bifurcation.
\end{abstract}

1. Introduction. Lattice Boltzmann schemes are a type of discrete algorithm which can be used to simulate fluid dynamics and more [3, 12, 32]. One of the nicest properties of an LB scheme is that the transport component of the algorithm, advection, is exact. All of the dissipation in the discrete system then occurs due to the relaxation operation. This dissipation occurs at different orders of the small parameter, the time step [28]. The first order gives an approximation to the Navier Stokes equations (with some error terms due to the discrete velocity system). The higher orders include higher space derivatives, similarly to Burnett and super-Burnett type systems. (They differ from the Burnett and super-Burnett terms and may have better stability properties, see a simple case study for the Ehrenfests' collisions in [24]). The form of the dissipative terms in the macroscopic dynamics is driven by the form of the discrete equilibrium, the analogy to the Maxwell distribution, the coefficients of the dissipative terms are selected by the collision operator. In the standard and most basic relaxation operation, the single time BGK relaxation [4], these coefficients cannot be modified independently. One mechanism which is used to stabilize LB schemes is to generalize this to an MRT operator where these coefficients can be varied independently [16, 17, 13, 14, 15, 25].

An MRT relaxation operation involves selecting a new basis to perform relaxation in. This basis must necessarily include the macroscopic moments (which do not vary during relaxation) and also includes elements used to vary the hydrodynamic viscosity. Depending on the size of the velocity set a number of degrees of freedom may remain available. These degrees of freedom can be used to modify the coefficients of some of the dissipative dynamics at the post Navier-Stokes level.

A barrier to the stability of certain LB schemes is the presence of parasitic oscillations in the vicinity of sharp gradients in the flow. These may be caused by shock waves travelling through the fluid as well as thin shear layers or singularities in boundary conditions. These oscillations are analagous to Gibbs oscillations and are common in discrete approximations. The MRT operation can be used to delete the contribution to these oscillations made by the higher order modes, by equilibrating them. The cost of this is a fixed increase in the dissipation coefficients in the post

${ }^{\dagger}$ Department of Mathematics, University of Leicester, Leicester, UK (\{ag153,dp153\}@le.ac.uk) 
Navier-Stokes dynamics.

Since most LB collisions are linear relaxations, linear stability of the collision operation is the most common minimal criteria for choice of the relaxation rate(s). One can however use a Lyapunov function instead and the physical meaning of such a function is an entropy function. There are two generic methods by which this technology is incorporated into LB systems.

A method to remove the dispersive oscillations in a single relaxation time setting, which makes use of these entropy functions, is Entropic Filtering [7, 8, 9]. Here the relaxation rate is not fixed across the system. Rather it can be varied in the presence of shock waves and oscillations to adaptively modify dissipation locally (including on the Navier-Stokes level). The choice of the local relaxation rate is informed by the change in the Entropy (Lyapunov) function given by different rates. Iin the presence of a shock wave, for example, a large contraction to equilibrium, with respect to the Lyapunov function, can be made. In particular the relaxation rate can be modified to implement a type of low pass filter, removing the non-physical high frequency oscillations while leaving the low frequence physical dynamics untouched. Filtering can also be applied at other stages of the lattice Boltzmann system, for example to the macroscopic dynamics [31].

In this work we attempt to combine the entropic filtering and MRT techniques by applying a type of filter to the separate modes of the MRT system. By doing so we hope to achieve the stability benefits exhibited by these methods while adding as little extra dissipation as possible.

An alternative suggested stabilizer is to use an entropic lattice Boltzmann method (ELBM) $[23,22]$. In this case the collision operator is modified to guarantee positive entropy production and hence respect the Second Law. In terms of stability this represents a complete replacement of the linear stability of collision operations, to a Lyapunov criteria for the chosen entropy function. In a single relaxation time setting this effect can be achieved be modifying the relaxation rate so that the limit of the relaxation is the so-called 'entropy balance'.

The difference between the ELBM and the entropic filtering comes in two parts. Firstly ELBM would be applied across the entire system, so the relaxation rate is different for every point in space. By comparison the entropic filtering should be applied at a small proportion ( $\ll 1 \%$ ) of the lattice sites which are far from equilibrium. Secondly the primary purpose of the ELBM is to guarantee the 'proper' entropy production for a particular $H$ function. From this some stability benefits may arise. To be particular one might say that it is hoped that Lyapunov stability of the collision, with respect to the particular entropy function, is a stronger requirement than the usual linear stability. The entropic filtering is designed entirely as a stability technique, some regions of the system may not satisfy the Second Law (for a particular $H$ function) just as in the BGK and MRT systems.

2. Single relaxation time $\mathbf{L B}$ schemes. The usual approach for deriving the single relaxation time LB systems starts from the Boltzmann Equation

$$
\partial_{t} f+\mathbf{v} \cdot \partial_{\mathbf{x}} f=Q(f)
$$

where $f \equiv f(\mathbf{x}, \mathbf{v}, t)$ is a one particle distribution function over space, velocity space and time and $Q(f)$ represents the interaction between particles, sometimes called a collision operation. The nonlinear Boltzmann collision integral $Q(f)$ is substituted by 
the Bhatnagar-Gross-Krook [4] equation

$$
Q(f)=-\frac{1}{\tau}\left(f-f^{\mathrm{eq}}\right)
$$

The BGK operation represents a relaxation towards the local equilibrium $f^{\text {eq }}$ with rate $1 / \tau$. The distribution $f^{\text {eq }}$ is given by the Maxwell Boltzmann distribution,

$$
f^{\mathrm{eq}}=\frac{\rho}{(2 \pi T)^{D / 2}} \exp \left(\frac{-(\mathbf{v}-\mathbf{u})^{2}}{2 T}\right)
$$

The macroscopic quantities are available as integrals over velocity space of the distribution function,

$$
\rho=\int f \mathrm{~d} \mathbf{v}, \quad \rho \mathbf{u}=\int \mathbf{v} f \mathrm{~d} \mathbf{v}, \frac{1}{2} \rho \mathbf{u}^{2}+\rho D \Theta=\frac{1}{2} \int \mathbf{v}^{2} f \mathrm{~d} \mathbf{v} .
$$

A quadrature approximation to these integrals is the first ingredient to discretize this system. The second is a time integration along the discrete velocities given by the quadrature. In order to achieve a fully discrete system it is necessary to make an appropriate choice of quadrature. The set of all quadrature nodes is denoted $\mathcal{V}=\left\{\mathbf{v}_{1}, \ldots, \mathbf{v}_{n}\right\}$. This set should be chosen such that it defines a discrete subgroup of space $\mathcal{L}$ called the lattice, which is invariant under shifts given by elements of $\mathcal{V}$, $\mathcal{L}=\mathcal{L}+\mathbf{v}_{i}$.

The scalar field of the population function (over space, velocity space and time) becomes a sequence of vector fields (over space) in time $f_{i}\left(\mathbf{x}, n_{t} \epsilon\right), n_{t} \in \mathbb{Z}$, where the elements of the vector each correspond with an element of the quadrature.

Explicitly the macroscopic moments are given by,

$$
\rho=\sum_{i=1}^{n} f_{i}, \quad \rho \mathbf{u}=\sum_{i=1}^{n} \mathbf{v}_{i} f_{i}, \quad \frac{1}{2}\left(\rho \mathbf{u}^{2}+\rho \Theta\right)=\frac{1}{2} \sum_{i=1}^{n} \mathbf{v}_{i}^{2} f_{i} .
$$

The complete discrete scheme is given by

$$
f_{i}\left(\mathbf{x}+\epsilon \mathbf{v}_{i}, t+\epsilon\right)=f_{i}(\mathbf{x}, t)+\omega\left(f_{i}^{\mathrm{eq}}(\mathbf{x}, t)-f_{i}(\mathbf{x}, t)\right)
$$

where $\epsilon$ is the time step and $\omega$ controls the degree of the relaxation in each individual step For this system a discrete equilibrium must be used. One way to find a discrete equilibrium is simply to evaluate a second order Mach number expansion of the Maxwell Boltzmann distribution at each quadrature node. The second order expansion is taken in order to guarantee the exact zero order hydrodynamics (the Euler equations). In the collision operation there is a new notation $\omega$ for the relaxation rate. This indicates that this parameter has a qualitatively different effect in the discrete system from the continuous one.

The relaxation coefficient $\omega$ depends on the continuous BGK relaxation time: $\omega=2 \epsilon /(2 \tau+\epsilon)$, and at first order in $\epsilon$, the viscosity $\nu=c_{s}^{2} \tau, c_{s}$ is the speed of sound. $\omega \in[1,2) . \omega=1(\tau=\epsilon / 2)$ corresponds to equilibrating at each time step and $\omega=2$ corresponds to a reflection and the zero viscosity limit.

3. MRT lattice Boltzmann. MRT has been proposed for LBM collision operation $[16,17,13,14,15,25]$, where the additional degrees of freedom arising from a velocity set with sufficient isotropy to reproduce the Navier-Stokes equations are exploited to introduce varying levels of dissipation on different modes of the system. 
Usually this involves a change of basis for the collision operation, the change of basis matrix can be denoted $M$. In this new basis a diagonal relaxation matrix $\Omega$ can be used to relax the different modes independently before the change of basis is reverted,

$$
\mathbf{f} \mapsto \mathbf{f}+M^{-1} \Omega M\left(\mathbf{f}^{\mathrm{eq}}-\mathbf{f}\right)
$$

The choice of which basis $M$ is 'best' is not obvious. Several components of the basis are necessary, inparticular the macroscopic moments should form a part of the basis. The next components of the basis should be chosen to represent the momentum flux in the system and may be subdivided to implement independent control of bulk and shear viscosities. The most interesting selection is the completion of the basis following this, corresponding to the post Navier-Stokes dynamics. The additional modes used to complete the basis may be known as 'ghost' modes.

To consider a choice of basis it is instructive to imagine the collision operation as a reduction in the non-equilibrium volume of a system. If for example we have a 9 velocity system with 3 conserved moments then this non-equilibrium volume is 6-dimensional. For the single relaxation time BGK operation this volume shrinks uniformly along any axis passing through the equilibrium. MRT allows us the possibility to shrink this volume independently along different axes, the choice of the MRT basis is equivalent to the choice of these axes. If the higher order modes are equilibrated at every time step then that component of the volume is removed entirely during any collision. When we are concerned with dynamically shrinking this volume however the choice of basis becomes especially important. We would like to choose a basis which will target the non-physical oscillations in the system.

One choice of basis which has been suggested is an $\ell_{2}$ orthogonal extension of the macroscopic moments made by cross powers of the velocity set $[17,25]$.

In this work we choose to use the basis used by Dellar [14] which has the alternative property of being orthogonal in the weighted inner product given by the quadrature weights. Our computational examples will be calculated on the D2Q9 lattice, this is a uniform nine-speed square lattice with horizontal and vertical components of the velocity set given by the vectors,

$$
\begin{aligned}
& v_{x_{1}}=(0,1,0,-1,0,1,-1,-1,1) \\
& v_{x_{2}}=(0,0,1,0,-1,1,1,-1,-1) .
\end{aligned}
$$

For the MRT implementation we will use the standard polynomial equilibrium,

$$
f_{i}^{\mathrm{eq}}=w_{i} \rho\left(1+3 \mathbf{v}_{i} \cdot \mathbf{u}+\frac{9}{2}\left(\mathbf{v}_{i} \cdot \mathbf{u}\right)^{2}-\frac{3}{2} \mathbf{u}^{2}\right) .
$$

Here, the lattice weights, $w_{i}$, are given lattice-specific constants: $w_{0}=4 / 9, w_{1,2,3,4}=$ $1 / 9$ and $w_{5,6,7,8}=1 / 36$. In addition to define the basis completely a supplementary vector is used,

$$
g=(1,-2,-2,-2,-2,4,4,4,4)
$$

this may be expressed in terms of the velocity set,

$$
g_{i}=1-\frac{15}{2}\left|\mathbf{v}_{i}\right|^{2}+\frac{9}{2}\left|\mathbf{v}_{i}\right|^{4} .
$$

Altogether then the change of basis matrix can be given by a matrix consisting of the 9 following rows, where just in this instance $I$ represents a row of ones, all vector 
multiplications are implied elementwise,

$$
M=\left[I ; v_{x_{1}} ; v_{x_{2}} ; v_{x_{1}}^{2}-I / 3 ; v_{x_{2}}^{2}-I / 3 ; v_{x_{1}} v_{x_{2}} ; g v_{x_{1}} ; g v_{x_{2}} ; g\right] .
$$

The first three modes in this basis correspond to the conserved moments and no relaxation is performed on these modes. In principle the other modes can be modified separately. In practice we use the same relaxation coefficient for the next three modes which together control bulk and shear viscosity. The next two modes are denoted $\mathbf{J}$ and could be modified to improve stability, in fact for these modes we just follow Dellar and use the same rate as the hydrodynamic modes. Our use of MRT then is limited to varying the relaxation rate of the final mode consisting of even order components, this ghost mode is denoted $N$.

In fact for a numerical implementation it is not necessary to explicitly form the complete change of basis operation. Again following Dellar, as the macroscopic moments are calculated we also calculate the ghost mode we are interested in modifying,

$$
N=\sum_{i=1}^{n} g_{i} f_{i}
$$

After that the MRT lattice Boltzmann can be implemented,

$$
f_{i}\left(\mathbf{x}+\epsilon \mathbf{v}_{i}, t+\epsilon\right)=f_{i}(\mathbf{x}, t)+\omega\left(f_{i}^{\mathrm{eq}}(\mathbf{x}, t)-f_{i}(\mathbf{x}, t)\right)-\left(\omega_{N}-\omega\right) w_{i} g_{i} N(\mathbf{x}, t) / 4 .
$$

Altogether then we have just two relaxation rate parameters, the standard rate $\omega$ and a further parameter separately controlling the relaxation of the ghost mode $\omega_{N}$. Evidently in the case $\omega=\omega_{N}$ this system reduces to the BGK collision operation.

A popular choice of rate for the ghost mode is to equilibrate it be setting $\omega_{N}=1$ and for the rest of this work doing so will be referred to as the 'standard' or 'usual' MRT operation. Since the equilibrium of this mode is zero this corresponds to deleting this component of the dynamics at each time step. This could be the most effective choice of parameter in terms of stability, however it may not be without penalty. Despite being orthogonal in the collision this mode is coupled to the hydrodynamics by means of the advection operation. In terms of the macroscopic dynamics this rate affects dissipation at a post Navier-Stokes level.

An alternative choice of rate for the ghost mode is to deliberately under relax the mode (set $\omega_{N}$ less than one). For the benchmark problem used in this work we find that the choice of $\omega_{N}=1$ outperforms this under-relaxation strategy, hence those results are not included. Some other realizations of the MRT lattice Boltzmann collisions were tested in [10].

4. Entropic collisions and the entropy balance. In the continuous case the Maxwellian distribution maximizes entropy, as measured by the Boltzmann $H$ function, and therefore also has zero entropy production. In the context of lattice Boltzmann methods a discrete form of the $H$-theorem has been suggested as a way to introduce thermodynamic control to the system [23].

From this perspective the goal is to find an equilibrium state equivalent to the Maxwellian in the continuum which will similarly maximize entropy. Before the equilibrium can be found an appropriate $H$ function must be known for a given lattice. These functions have been constructed in a lattice dependent fashion in [22]. The general form of the perfect lattice Boltzmann entropy is

$$
S(\mathbf{f})=-\sum_{i} f_{i} \log \left(\frac{f_{i}}{w_{i}}\right) .
$$


Here, the $w_{i}$, are the lattice weights. The celebrated example gives us the D2Q9 lattice $\left(w_{0}=4 / 9, w_{1,2,3,4}=1 / 9\right.$ and $\left.w_{5,6,7,8}=1 / 36\right)$. The entropic equilibrium state, $f^{\mathrm{eq}}$, is uniquely determined by maximizing the entropy functional (4.1) subject to the constraints of conservation of mass and momentum [2]:

$$
\sum_{i} f_{i}=\rho \quad \sum_{i} \mathbf{v}_{i} f_{i}=\rho \mathbf{u} .
$$

The equilibrium which maximizes this is also known explicitly,

$$
f_{i}^{\mathrm{eq}}=w_{i} \rho \prod_{j=1}^{2}\left(2-\sqrt{1+3 u_{j}^{2}}\right)\left(\frac{2 u_{j}+\sqrt{1+3 u_{j}^{2}}}{1-u_{j}}\right)^{v_{i, j}} .
$$

The entropic equilibria should maximize the entropy and, at the same time, it is expected that the LBGK scheme (2.6) with these equilibria approximates the proper transport equation. This combination of conditions may be too strong for the existence of such equilibria, especially, if one adds additional restrictions like polynomial form of the discrete equilibria [33, 34]. In addition, some restrictions on the Mach number are needed for the discrete $H$-theorem [34].

With the entropic equilibrium, the entropic collisions with exact balance of entropy are proposed. Together they form ELBGK [1]. In this case the single relaxation time is varied to ensure a constant entropy condition according to the discrete $H$ theorem. This can be implemented by implementing an extra relaxation parameter into the BGK collision, this can be called the EBGK collision,

$$
f_{i} \mapsto f_{i}+\frac{\omega \alpha}{2}\left(f_{i}^{\mathrm{eq}}-f_{i}\right)
$$

Here $\alpha$ effectively replaces the mirror reflection in the equilibrium as the limit of the over-relaxation. The parameter $\alpha$ should be selected as the non-trivial root of the equation

$$
S(\mathbf{f})=S\left(\mathbf{f}+\alpha\left(\mathbf{f}^{\mathrm{eq}}-\mathbf{f}\right)\right)
$$

ELBGK finds the value of $\alpha$ that with $\omega=2$ (inviscid fluid) would give zero entropy production, therefore making the position of zero entropy production the limit of any relaxation. For the fixed $\alpha$ used in the LBGK method it remains possible, particularly for low viscosity fluids, to relax past this point resulting in negative entropy production, violating the Second Law.

The ELBGK collision obviously respects the Second Law (if $\omega \leq 2$ ), and a simple analysis of entropy production gives the proper evaluation of viscosity.

The splitting of the entropic collision in two steps, entropic involution and linear contraction (4.4), assumes that both steps are possible. The entropic involution is not always possible: for highly nonequilibrium states $\mathbf{f}$ equation (4.5) may have no nonzero solution. Strictly speaking, the existence of the entropic involution is necessary for existence of collisions with preservation of entropy and it is sufficient (but not necessary) for existence of collisions with given entropy production. It is possible to relax the requirement of the existence of the entropic involution and to introduce entropic contraction. For this purpose, let us introduce a different form of the ELBGK collision operation which we call enhanced entropic collisions (EEC). We directly 
search for the distribution function $\mathbf{f}^{\prime}=\mathbf{f}+\alpha\left(\mathbf{f}^{\text {eq }}-\mathbf{f}\right)(\alpha \geq 1)$ which satisfies the equation $(1 \leq \omega<2)$,

$$
S\left(\mathbf{f}^{\prime}\right)-S\left(\mathbf{f}^{\mathrm{eq}}\right)=\sqrt{\omega-1}\left(S(\mathbf{f})-S\left(\mathbf{f}^{\mathrm{eq}}\right)\right) .
$$

If $\omega$ is close to 2 then EEC approaches ELBGK collisions. If $\mathbf{f}$ is close to $\mathbf{f}^{\text {eq }}$ then both EEC and ELBGK coincide with the simple LBGK in the main order. If $\omega$ is close to 1 then EEC coincides with LBGK. This is not true for ELBGK because the nontrivial solution to (4.5) may not exist. Its existence does not depend on $\omega$ but only on the populations. Attempting to solve (4.5) assumes that the entropic reflection exists, which is a strong requirement of existence of collisions with preservation of entropy whereas we just need a proper entropy balance.

4.1. Existence and non-existence of entropy balance. In [20] it was demonstrated that near an equilibrium there always exists a vicinity where it is possible to introduce LBM collisions with the proper entropy balance. At the same time it was proven, that for the standard Boltzmann type entropy function used in this work, there always exist areas of nonequilibrium distributions where the LBM collisions with the proper entropy balance are impossible. In particular the simplex of distributions can be split into two subsets $A$ and $B$ : in the set $A$ the entropic involution exists, and for distributions from the set $B$ equation (4.5) has no non-trivial solutions. Both sets $A$ and $B$ have non-empty interior (apart from a trivial symmetric degenerated case). In the numerical experiments conducted later in this work this scenario did not arise.

4.2. Numerical solution of entropy balance. In order to numerically investigate the stabilization properties of ELBGK it is necessary to use a numerical method capable of finding the non-trivial root in (4.5), while remaining as computationally efficient as possible. For a given lattice site and time step we recast Eq (4.5) as a function of $\alpha$ only:

$$
F(\alpha)=S\left(\mathbf{f}+\alpha\left(\mathbf{f}^{\mathrm{eq}}-\mathbf{f}\right)\right)-S(\mathbf{f}) .
$$

In this setting we attempt to find the non-trivial root $r$ of (4.7) such that $F(r)=0$. It should be noted that as we search for $r$ numerically we should always take care that the approximation we use is less than $r$ itself. An upper approximation could result in negative entropy production.

The algorithm we use in this work is simple Newton iterations, because of the concavity of $S$ the accuracy of the $n$th iteration of the method can be estimated,

$$
\left|\alpha_{n}-r\right| \lesssim\left|F\left(\alpha_{n}\right) / F^{\prime}\left(\alpha_{n}\right)\right|
$$

In fact we use a convergence criteria based not solely on $\alpha$ but on $\alpha\left\|\mathbf{f}^{\text {eq }}-\mathbf{f}\right\|$, this has the intuitive appeal that in the case where the populations are close to the local equilibrium $\Delta S=S\left(\mathbf{f}^{\mathrm{eq}}\right)-S(\mathbf{f})$ will be small and a very precise estimate of $\alpha$ is unnecessary.

Altogether we stop the method at the point,

$$
\left|\alpha_{n}-r\right| \cdot|| \mathbf{f}^{\mathrm{eq}}-\mathbf{f}||<\epsilon_{\mathrm{ELBM}}
$$

To ensure that we use an estimate that is less than the root, at the point where the method has converged we check the sign of $F\left(\alpha_{n}\right)$. If $F\left(\alpha_{n}\right)>0$ then we have 
achieved a lower estimate, if $F\left(\alpha_{n}\right)<0$ we correct the estimate to the other side of the root with a double length Newton step,

$$
\alpha_{n}=\alpha_{n}-2 \frac{F\left(\alpha_{n}\right)}{F^{\prime}\left(\alpha_{n}\right)} .
$$

At each time step before we begin root finding we eliminate all sites with $\Delta S<$ $10^{-15}$. For these sites we make a simple LBGK step. At such sites we find that round off error in the calculation of $F$ by solution of equation (4.7) can result in the root of the parabola becoming imaginary. In such cases a mirror image given by LBGK is effectively indistinct from the exact ELBGK collision.

In the numerical experiments in this work the chosen value of $\epsilon_{\mathrm{ELBM}}$ is $10^{-5}$, due to the fast convergence of this method we anecdotally observe that the actual accuracy reached is roughly of the order $10^{-10}$.

We implement ELBM in the form 'as it is described'. For this purpose, we estimate the nontrivial root $\alpha$ of the equation (4.5) from below with high accuracy. Then we use the entropic collision (4.4) with the same $\omega$ as for LBGK and other methods and compare the results. In some publications, a regularization by ELBM was reported that cannot be reproduced in such a way (compare, for example [1] to [27]). This may be caused, for example, by additional smoothing operations implemented in [1] and similar papers but omitted in the reports.

5. Oscillations and filtering. Dispersive oscillations pose a problem for low viscosity (high Reynolds number) flows in certain types of lattice Boltzmann simulations. In particular non-physical oscillations arise near to sharp gradients and singularities. These oscillations may cause the system to 'blow up' or simply pollute the system enough to render the results useless. We can use a signal filtering technique to target and remove these oscillations from the system.

5.1. Entropic filtering. Entropic filtering is a technique by which the relaxation rate in a BGK system is manipulated locally in order to suppress dispersive oscillations. The entropic terminology comes from using a discrete entropy function as an indicator for an unstable region of the fluid. We can say that entropic filtering uses some of the technology of the ELBM, but with a rather different motivation. In ELBM the entropy function is used as a Lyapunov function for the collision operation. Collisions are modified so that across the system positive entropy is produced with respect to the chosen entropy function. In entropic limiting the same entropy functions are used, but only as a measure of distance from equilibrium. The purpose of measuring this distance is to introduce an additional contraction if it becomes too much. Since entropic filtering is only applied at a small number of lattice sites, positive entropy production is not guaranteed across the system.

It has been shown that discrete entropy functions may be constructed in a lattice dependent fashion. These functions can be used in an Entropic Lattice Boltzmann Method (ELBM) [23, 22] as a Lyapunov function, which under certain conditions can be used to derive stability theorems. For these techniques we simply use a discrete entropy function as a metric for 'distance from equilibrium'. There are a number of other intuitively reasonable choices of metric, such as the 1 norm $\left\|\mathbf{f}-\mathbf{f}^{\mathrm{eq}}\right\|_{1}$, to do the same job but in this work we use the so called relative non-equilibrium entropy [8], this entropy function can be used with standard polynomial equilibria,

$$
\Delta S=\sum_{i} f_{i} \log \left(\frac{f_{i}}{f_{i}^{\mathrm{eq}}}\right) .
$$


A useful approximation to this $\Delta S$ gives the quadratic entropy (the second order Taylor expansion):

$$
\Delta S=\sum_{i} \frac{\left(f_{i}-f_{i}^{\mathrm{eq}}\right)^{2}}{f_{i}^{\mathrm{eq}}} .
$$

This formula can be used without positivity control: it is not singular for negative $f_{i}$.

We use the logic that any lattice site which exhibits very high non-equilibrium entropy, above a certain threshold, represents the peak of a dispersive oscillation. At such a site it could be appropriate to modify the relaxation rate to bring the distribution function back near to equilibrium, and thereby erase the peak of the oscillation. One option to stabilize the system is to return such lattice sites directly to their local equilibrium in an 'Ehrenfest Step' If we prefer to maintain the low frequency features of the non-equilibrium dynamics then the equivalent of a low pass filter can be applied.

A filter which has successfully been applied to single relaxation time lattice Boltzmann systems is the median filter [8]. This can be implemented as a post-process to a relaxation operation. A filter is formed of some computational molecule of nearby neighbours. In this work where we use the D2Q9 system we use the set of adjacent non-boundary lattice sites. The median average of the non-equilibrium entropy $\Delta S_{m e d}$ and the scaling coefficent $\delta=\sqrt{\Delta S_{\text {med }} / \Delta S}$ are then calculated. A site with high non-equilibrium entropy can then be updated again,

$$
f_{i} \mapsto f_{i}^{\mathrm{eq}}+\delta\left(f_{i}-f_{i}^{\mathrm{eq}}\right) .
$$

The coefficient $\delta$ arises from the quadratic approximation to the discrete entropy function and collapses the non-equilibrium volume of the filtered site back to the level (in non-equilibrium entropy terms) of the median of its neighbours.

The reason for selecting the median filter to regularize the lattice Boltzmann system is that it preserves the low frequency dynamics while eliminating the high frequency parts.

5.2. Median filtering MRT. We are now interested in implementing a median filter for the separate modes of the MRT collision operation. In an MRT setting our post-processing operation is given by,

$$
f_{i} \mapsto f_{i}^{\text {eq }}+\delta\left(f_{i}-f_{i}^{\text {eq }}\right)+\left(\delta_{N}-\delta\right) w_{i} g_{i} N / 4 .
$$

Evidently in the case $\delta=\delta_{N}$ this reduces to the single relaxation time limiter.

In this setting we have two parameters we may wish to manipulate, $\delta$ and $\delta_{N}$, and there are a few ideas we can present as to how to utilize them;

1. We can use the standard BGK collision across the system, set $\delta=1$ and dynamically choose $\delta_{N}$. This corresponds to not filtering the Navier-Stokes level modes at all, we attempt to locally trim out the oscillations in the ghost mode $N$. This is a weaker operation than using a full MRT (which would be equivalent to setting $\delta_{N}=0$ everywhere) but we hope it will be nearly as effective while adding less dissipation.

2. We can use the full MRT collision everywhere and dynamically choose $\delta$. This corresponds to equilibrating the ghost mode $N$ and trimming out oscillations in the other dynamics. This is a stronger operation than the usual MRT and might be appropriate for use at Reynolds numbers where the standard MRT fails to stabilize the system. It also adds dissipation on the Navier-Stokes and J moments. 
3. We can use the BGK collision everywhere and dynamically and separately choose both $\delta$ and $\delta_{N}$. The rationale for doing so is that we filter all the modes as in the single relaxation time setting, but due to the extra freedom in parameters we can treat separate modes independently. Therefore if oscillations appearing in different modes can be targetted for deletion separately, without adding undue dissipation on all modes.

As $\delta_{N}$ controls the dissipation on a single mode it is relatively straightforward to create a strategy to select a value for it. We simply take the ratio of absolute value of the mode to the median absolute value of its neighbours, $\delta_{N}=|N| /|N|_{\text {med }}$ where $|N|_{\text {med }}$ is the median of the absolute values of $N$ locally. It is also simple to select which sites to apply the filter at, we simply take the sites where $|N|$ is above a certain threshold.

Selecting a relaxation for the combined remaining nodes is not so obvious as again there are a number of reasonable choices. We use the quadratic approximation to the non-equilibrium entropy of the populations with the contribution due to $N$ moment stripped out, we can define an auxiliary set of populations,

$$
f_{i}^{*}=f_{i}-w_{i} g_{i} N / 4
$$

The non-equilibrium entropy of this component of the dynamics is evaluated by the quadratic approximation of the relative entropy (5.2). The coefficient $\delta$ may be calculated as $\delta=\sqrt{\Delta S_{\text {med }} / \Delta S}$, where again $\Delta S_{\text {med }}$ is the median value of $\Delta S(5.2)$ locally.

Due to the differing methods for calculating the post processing coefficients $\delta$ and $\delta_{N}$, as well as the relative size of the modes, it is not appropriate to use the same threshold for selecting filtering sites. A separate threshold should be chosen for each, which selects a small proportion of the sites for filtering.

6. Numerical tests. In this section we test the effectiveness of the techniques detailed above at stabilizing the lattice Boltzmann method, and at what cost.

The numerical benchmark that we use is the lid-driven cavity. This is a $2 \mathrm{D}$ test problem which consists of a square domain with a tangential velocity applied at the top boundary edge. This boundary condition drives the formation of vortices, which for low Reynolds numbers form a steady state and, for high Reynolds numbers, periodic or chaotic results. This transition begins with a Hopf bifurcation where the steady state evolves into a periodic flow, and localizing this bifurcation will be our goal. The lid-driven cavity has already been used for lattice Boltzmann methods [21], including in particular for the comparison of collision operations [26]. In these works the accuracy of the steady states pre-bifurcation were considered whereas now we are concerned with the onset of periodicity.

In this study we use diffusive boundary conditions. The essence of the condition is that populations reaching a boundary are reflected, proportional to equilibrium, such that mass-balance (in the bulk) and detailed-balance are achieved. In each corner of the cavity, similarly to in the bounce-back boundary condition the outward population is simply reflected inward to conserve mass.

To illustrate, immediately following the advection of populations consider the situation of a wall, aligned with the lattice, moving with tangential velocity $u_{\text {wall }}$ and with inward normal in the down direction. The implementation of the diffusive Maxwell boundary condition at a boundary site $\mathbf{x}$ on this wall consists of the update

$$
f_{i}(\mathbf{x}, t+1)=\gamma f_{i}^{\mathrm{eq}}\left(u_{\text {wall }}\right), \quad i=4,7,8,
$$


TABLE 6.1

Results of lid driven cavity simulations

\begin{tabular}{|c|c|c|c|c|}
\hline Paper & Method & $\mathrm{Re}$ & $\begin{array}{l}\text { Grid } \\
\text { Size }\end{array}$ & $\begin{array}{l}\text { Re of } \\
\mathrm{HB}^{*}\end{array}$ \\
\hline Brezillon et al [6] & $\begin{array}{l}\mathrm{FE}^{*}, 12 \text { modes and } 13122 \\
\text { DoF* }\end{array}$ & $\begin{array}{l}6000- \\
11000\end{array}$ & $\mathrm{~N} / \mathrm{A}$ & 7890 \\
\hline Brownlee et al [9] & Median Filtered LBM & $0-8000$ & 100 & 7135 \\
\hline $\begin{array}{l}\text { Bruneau \& Saad } \\
{[11]}\end{array}$ & $\begin{array}{l}\text { Mixed order (3rd and } \\
\text { 2nd) FD* scheme }\end{array}$ & $\begin{array}{l}10- \\
10000\end{array}$ & $\begin{array}{l}256- \\
1024\end{array}$ & $\begin{array}{l}8000- \\
8050\end{array}$ \\
\hline Hou et al[21] & BGK type LBM & $10-7500$ & 256 & $\mathrm{~N} / \mathrm{A}$ \\
\hline Luo et al $[26]$ & MRT type LBMs & $\begin{array}{l}100- \\
1000\end{array}$ & $\begin{array}{l}65- \\
513 \\
\end{array}$ & $\mathrm{~N} / \mathrm{A}$ \\
\hline Peng et al [30] & $\begin{array}{l}\text { Mixed order ( } 7 \text { th and } \\
6 \text { th) FD scheme }\end{array}$ & $0-10500$ & $\begin{array}{l}100 \\
150 \\
200\end{array}$ & $\begin{array}{l}7402 \\
7694 \\
7704\end{array}$ \\
\hline
\end{tabular}

with

$$
\gamma=\frac{f_{2}(\mathbf{x}, t)+f_{5}(\mathbf{x}, t)+f_{6}(\mathbf{x}, t)}{f_{4}^{\mathrm{eq}}\left(u_{\text {wall }}\right)+f_{7}^{\mathrm{eq}}\left(u_{\text {wall }}\right)+f_{8}^{\mathrm{eq}}\left(u_{\text {wall }}\right)} .
$$

The reason for the selection of this boundary condition vs the often used and standard 'bounce-back' condition, is that this choice results in reduced noise in the populations functions and therefore the macroscopic moments. When using the bounce-back boundary conditions this noise makes the transition from a steady to a periodic flow difficult to detect.

A survey of available literature reveals that the precise value of Re at which the first Hopf bifurcation occurs is somewhat contentious, with most current studies (all of which are for incompressible flow) putting the bifurcation in the range $\mathrm{Re}=7400$ $8500[11,29,30]$. Here, we do not intend to give a precise value, rather we will be content to approximately localise the first bifurcation and by doing so measure the relative amount of dissipation produced by different systems. A system which becomes unsteady before a Reynolds number in this range is achieved can be considered to be unstable at that point. In 6.1 we detail some results of lid driven cavity simulations.

To localise the first bifurcation we take the following algorithmic approach. The initial uniform fluid density profile is $\rho=1.0$ and the velocity of the lid is $u_{\text {wall }}=1 / 10$ (in lattice units). This is chosen as it is a typical lid speed used in previous works $[21,25]$, similar experiments were carried out for $u_{\text {wall }}=1 / 100$ and no qualitative differences in the relative performances of the methods were observed. We use 101 interior lattice points in each direction for the domain. The equilibria used are the entropic equilibria (Eq. 4.3) for ELBGK and polynomial equilibria (Eq. 3.3) for all other systems. We record the velocity data at a single control point with coordinates $(6,18)$ (relative to the upper left corner) and run the simulation for $5000 L / u_{0}$ time steps. We denote the final $0.1 \%$ of the time series given by this signal by $\mathbf{u}_{\text {sig }}$.

In this problem the standard BGK system becomes unsteady before the expected Reynolds number of the first bifurcation, at approximately $R e=2500$. For our next benchmark test we examine the bifurcation in the standard MRT system. 

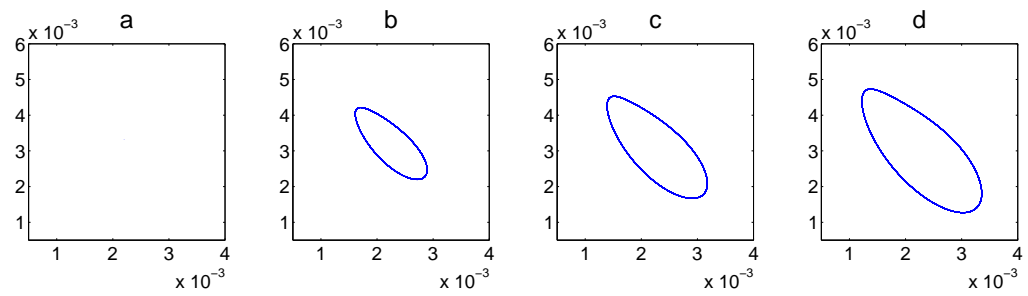

Fig. 6.1. Mean filtered $\mathbf{u}_{\text {sig }}$ for the standard $M R T$ at a) $R e=7400$, b) $R e=7600$, c) $R e=7800$, d) $R e=8000$

In Figure 6.1 the emergence of a periodic state in the standard MRT system is observed. Again we do not precisely locate the bifurcation, only noting that it appears to emerge between a steady state solution at $\mathrm{Re}=7400$ and a periodic solution at $\mathrm{Re}=7600$. We should note that any additional dissipation produced by this operation has an extremely limited effect as the first bifurcation is detected in the expected region.
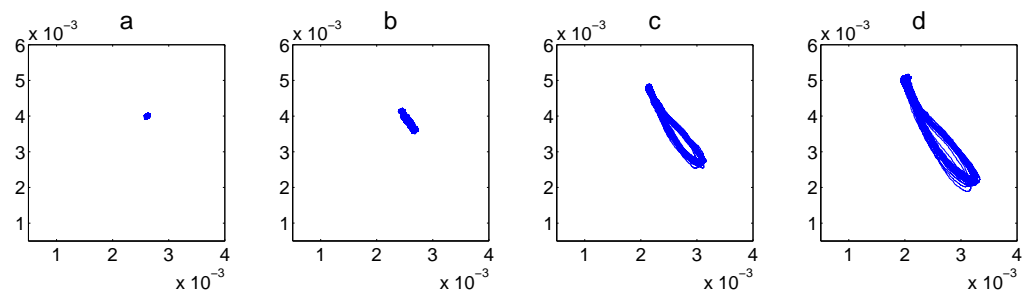

FIG. 6.2. Mean filtered $\mathbf{u}_{\text {sig }}$ for the BGK system equipped with the single relaxation time median filter at a) $R e=7400$, b) $R e=7600$, c) $R e=7800$, d) $R e=8000$

The next benchmark is the BGK system with the single relaxation time Median Filter. In Figure 6.2 we see that this system similarly seems to pick a bifurcation between $\operatorname{Re}=7400$ and $\operatorname{Re}=7600$, although the form of that bifurcation is somewhat different and the system is subject to some noise due to the non-constant application of the limiter.

The final benchmark is the test of the ELBM system. In this experiment the system becomes unsteady before the expected Reynolds number of the first bifurcation at approximately $\mathrm{Re}=6700$.

The next test involves filtering only the $N$ mode as discussed previously. In this case we set a threshold of $10^{-2}$ for sites where the filter is applied. In particular, we make two tests where we observe the following:

- In a test where the relaxation rate $\delta_{N}$ is selected dynamically the system becomes unsteady before the expected Reynolds number of the first bifurcation at approximately $R e=3800$. In this instance approximately 23 lattice sites per time step are selected for filtering.

- In a test where the relaxation rate $\delta_{N}$ is always chosen as zero the system becomes unsteady before the expected Reynolds number of the first bifurcation 
at approximately $\mathrm{Re}=4700$. In this instance approximately 20 lattice sites per time step are selected for filtering.

Altogether we observe that applying an MRT operation in this manner is considerably less effective at stabilizing the system than the standard method of applying it uniformly everywhere in the system.

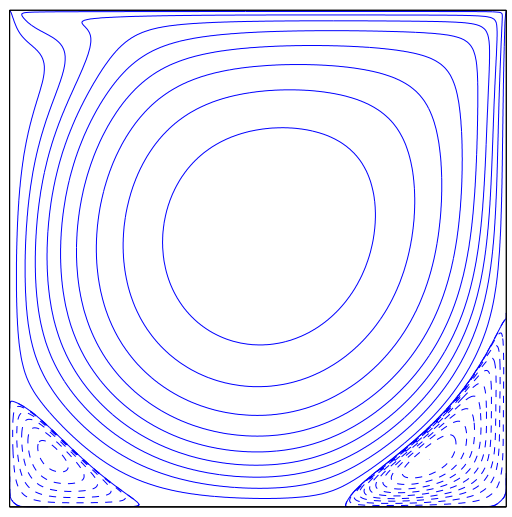

FIG. 6.3. Stream function of BGK with an enlarged grid size of 401 at the final time step of the $R e=7000$ simulations (negative stream regions in dashed lines)

a

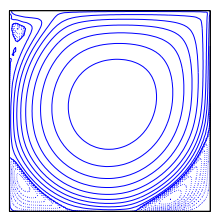

b

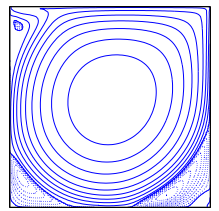

C

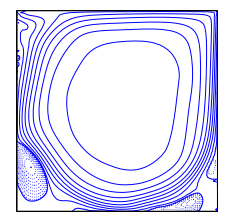

d

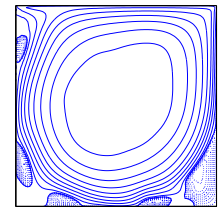

FIG. 6.4. Stream function of a) Full $M R T$, b) BGK equipped with the single relaxation time median filter c) BGK with MRT Limiter with $\delta_{N}$ dynamically chosen, d) ELBM with the standard grid size of 401 at the final time step of the Re=7000 simulations (negative stream regions in dashed lines)

Although ELBM and these MRT-filtered systems become unsteady before the expected Reynolds number of the first bifurcation, the systems may complete the requested number of time steps (they do not 'blow up') but in fact complete the simulation up to higher values of Reynolds numbers. Nevertheless the results of these simulations are not necessarily useful as can be seen in Figures 6.3,6.4. The large panel given in Figure 6.3 is the standard BGK system on a much larger grid size $(401 \times 401)$ so that the system is steady up to Re7000. The first two panels of Figure 6.4 are qualitatively similar, with the caveat that the larger grid introduces some extra stability, accounting for the disappearance of the small sub vortex in the upper left corner. The form of the stream function in the remaining two panels varies 
significantly from the large grid system.
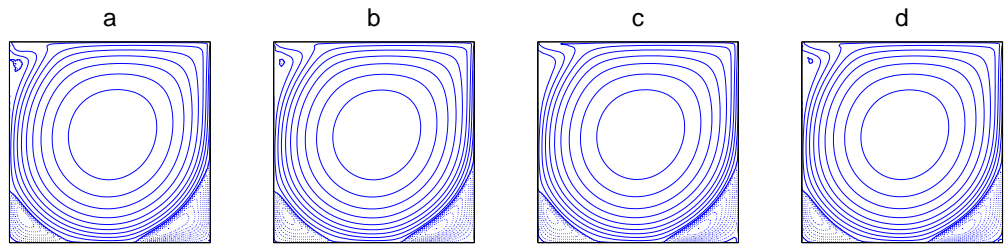

FIG. 6.5. Steam function of a)BGK, b) Full MRT, c) BGK equipped with the single relaxation time median filter, d) ELBM at the final time step of the Re=5000 simulations (negative stream regions in dashed lines)

The other side of the coin is that although simulations may not be completely steady before the bifurcation, the results may be quite good up to higher values of Re. In Figure 6.5 the stream functions are given for all the benchmark tests at $R e=5000$. For this Reynolds number BGK (a) is unsteady (having non-zero disturbance energy), nevertheless the stream function is rather close to the steady solutions given by the three primary stabilizing techniques and positions of the vortex centres are practically stationary. We presume that in this case the disturbances in the pure BGK systems consist of minor fluctuations which average out in time. This explains the good results for vortex centres for BGK at this Reynolds number and grid resolution given elsewhere [9].
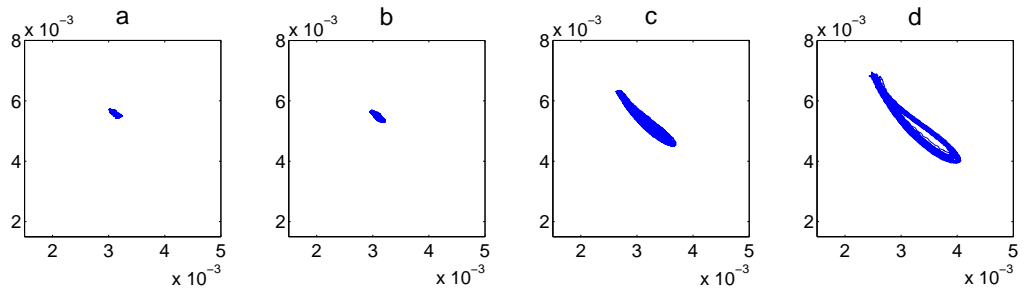

FIG. 6.6. Mean filtered $\mathbf{u}_{\text {sig }}$ for the full MRT system equipped with the median filter as a post-processor at a) $R e=7400$, b) $R e=7600$, c) $R e=7800$, d) $R e=8000$

The next test represents using the full MRT everywhere and applying the Median Filter as a post-processor. We can see from Figure 6.6 that although this system also picks out the first bifurcation, it does so at a larger Reynolds number.

The final techniques to test are the combinations of filtering both parameters $\delta$ and $\delta_{N}$. We try two options, in both for the Navier-Stokes part we use the Median Filtering where $\Delta S$ is above the threshold. For the $N$ component in the first option we dynamically choose $\delta_{N}$, in the second option we just set $\delta_{N}=0$, where the magnitude of $N$ exceeds the threshold.

In Figures 6.7, 6.8 we see the systems with the median-filtered Navier Stokes dynamics as well as the median filtered/ selectively equilibrated ghost mode respectively. In these systems we see the bifurcation develops into a very noisy phase before 

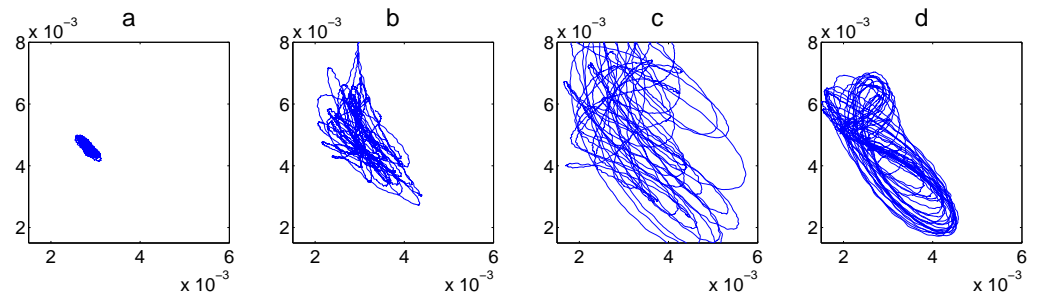

FIG. 6.7. Mean filtered $\mathbf{u}_{\text {sig }}$ for the BGK system with the Navier-Stokes modes and ghost mode median filters as a post-processor at a) $R e=7400, b) R e=7600$, c) $R e=7800$, d) $R e=8000$
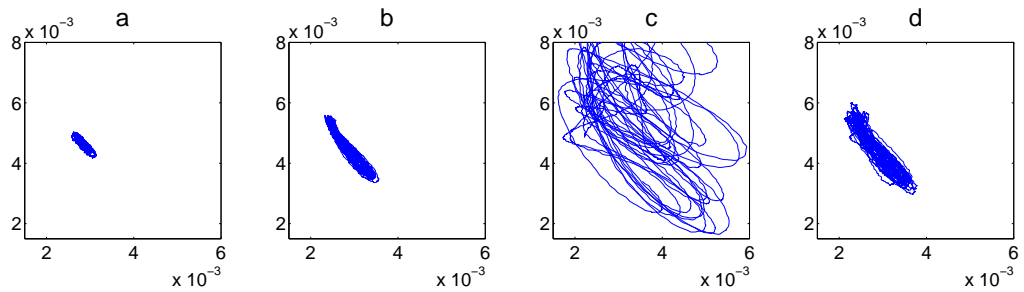

FIG. 6.8. Mean filtered $\mathbf{u}_{\mathrm{sig}}$ for the BGK system with the Navier-Stokes modes and ghost mode selectively equilibrated as a post-processor at a) $R e=7400$, b) $R e=7600$, c) $R e=7800$, d) $R e=8000$

seeming to return to a more structured dynamic.

To further examine the relative positions of the bifrucations in the systems we measure the mean squared deviation of $\mathbf{u}_{\text {sig }}$ from its own average in that time, this gives the 'disturbance energy' of the signal,

$$
E=\left\|\left(u_{\text {sig }}-\overline{u_{\text {sig }}}\right)\right\|_{2}^{2} .
$$

For the steady state $E=0$ and post bifurcation $E \propto\left(R e-R e_{c}\right)$ where $R e_{c}$ is the Reynolds number of the bifurcation.

In Figure 6.9 we plot $E$ as a function of Reynolds number, including the results of the benchmark tests. The emergence of the first bifurcation in the systems shown in Figures 6.1, 6.2,6.6, 6.7, 6.8 can also be observed here. From this figure we can see that the standard MRT as well as the two limiters which filter the modes independently produce marginally less dissipation.

In Figure 6.10 we extend the range of Reynolds numbers up to 100000. The scope of energy exhibited by the systems diverges apart perhaps from the full MRT and the full MRT with Median Filter which stay relatively close together until the full MRT becomes unstable at approximately $\mathrm{Re}=44000$.

7. Conclusion. The first observation is that in both the MRT and Median Filtered simulations the first bifurcation is detected in the expected range. We should not say that the bifurcation appears at the proper value, as we do not have a precise expectation of what it should be. Rather we can just say that both techniques add a minor and comparable amount of dissipation into the system, while improving the stability relative to the BGK system. 

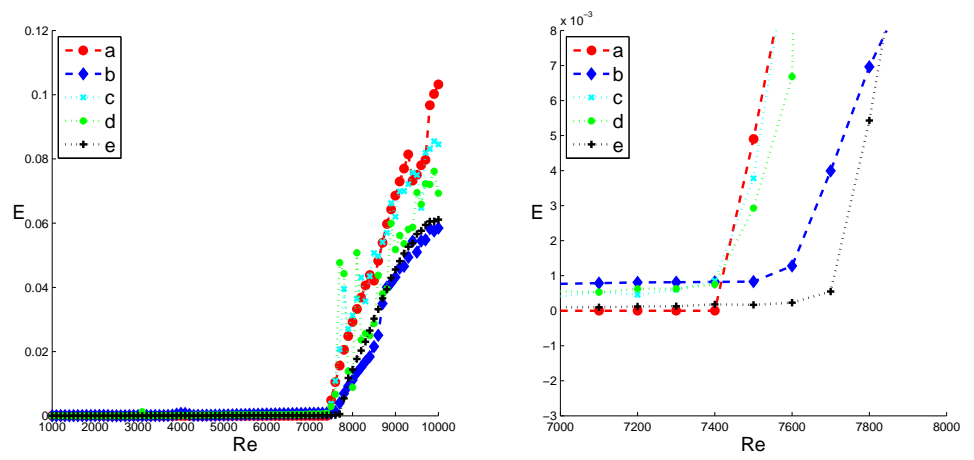

FIG. 6.9. Disturbance energy (Eq. 6.3) in the systems with collision operations a) Full MRT b) $B G K$ with single relaxation time Median Filtering c) BGK with Median Filtering the NavierStokes and ghost modes independently d) BGK with Median Filtering the Navier-Stokes modes and selectively equilibrating the ghost mode e) Full MRT with Median Filtering the Navier-Stokes modes. Plots represented for two different scales: at $R e=1000$ to $R e=10000$ and at $R e=7000$ to $R e=8000$

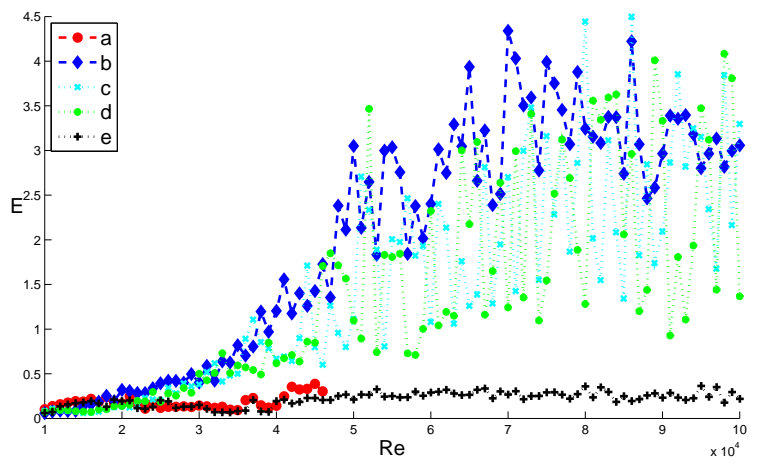

FIG. 6.10. Disturbance energy (Eq. 6.3) in the systems with collision operations a) Full MRT b) $B G K$ with single relaxation time Median Filtering c) $B G K$ with Median Filtering the NavierStokes and ghost modes independently d) BGK with Median Filtering the Navier-Stokes modes and selectively equilibrating the ghost mode e) Full MRT with Median Filtering the Navier-Stokes modes; at $\operatorname{Re}=10000$ to $\operatorname{Re}=100000$

The other primary technique outlined in this work, ELBGK, stabilized the system up to a higher Reynolds number than the basic LBGK system, however not enough to reach the first bifurcation. On the basis of this test this technique cannot be recommended as a stabilizer for lattice Boltzmann systems as it not only performs worse, but is significantly more computationally expensive than an application of MRT or Median Filtering, due to the requirement to solve Eq 4.7 at every lattice site and time step. Low levels of stabilizing performance have also been found for other benchmark problems [27]. This seems very natural in the light of the analysis of entropic methods in other areas. Precise entropy balance, for example, does not decrease the dispersive oscillations in the finite difference simulations [35].

The experiments which only filtered the ghost mode $N$ were also not stable up to the first bifurcation. While it may be interesting that such minor modifications to the BGK system (altering only some of the post Navier-Stokes dynamics at a small number of sites) provides a stability increase by $R e=1000-2000$, on the basis 
of this test this limiter cannot be recommended very strongly. The reasons being that the standard MRT is considerably more effective, exhibits a reasonable value for the first bifurcation (and hence adds little dissipative error) and doesn't bear the computational overhead of applying a post-processing operation in addition to evaluating the field $N$ at each time step.

The combination of the full MRT and the Median Filter as a post process introduces an additional quantity of dissipation which delays the onset of the first bifurcation, as might be expected as the combination of two smoothing techniques. This combination could be appropriate for applications where the stabilizing effects of the MRT alone are not sufficient.

The systems we tested which attempted to filter the modes independently also stabilize the system, picking a reasonable value of the Reynolds number for the first bifurcation but with some additional noise in the dynamics.

According to Figure 6.9 the systems which pick the bifurcation at the lowest value of Re are the Full MRT system and the two systems which filter the modes independently, although the difference is quite marginal. All the results of the filtered systems will be affected by the particular choice of the thresholds used to select the sites where filtering is applied. Fewer applications of any filter would be expected to move the bifurcation to a lower Reynolds number. No investigation was made in this work into varying these thresholds, they were simply chosen via some initial tests as values which selected a very small proportion of lattice sites.

We also observe that in the realm of higher Reynolds numbers (Figure 6.10) that the energy of the systems at the monitoring point diverges. Another method of validation should be used at these lower viscosities to realize which technique is most accurate in this regime.

Altogether we have observed that two existing techniques for stabilizing lattice Boltzmann methods by generalizing the BGK collision operation succeed in picking the first Hopf Birfucation at a reasonable value of the Reynolds number. Of course these two operations can be combined to produce even more artificial dissipation and hence move the bifurcation to a greater value of the Reynolds number. This is obviously undesirable but such a procedure may be useful at very low viscosities to produce a stable system. Another existing technique, ELBM, performs more poorly, providing less stability benefits while being more computationally expensive. The newly created limiters, which filter the modes independently, also pick a reasonable of the Reynolds number for the first bifurcation. A fuller investigation into the effects of altering the threshold parameters could to be made to see if this performance can be further improved. The fact that the 'parent' methods of these limiters, namely MRT and Median Filtering, perform so well, indicates that any performance gains from using a limiter of this type will be fairly marginal.

\section{REFERENCES}

[1] S. Ansumali and I. V. Karlin, Stabilization of the lattice Boltzmann method by the $H$ theorem: A numerical test, Phys. Rev. E, 62 (2000), pp. 7999-8003.

[2] S. Ansumali S, I.V. Karlin, and H.C. Ottinger, Minimal entropic kinetic models for hydrodynamics, Europhys. Lett., 63 (2003), pp. 798-804.

[3] R. Benzi, S. Succi, and M. Vergassola, The lattice Boltzmann equation: theory and applications, Physics Reports, 222 (1992), pp. 145 - 197.

[4] P. L. Bhatnagar, E. P. Gross, and M. Krook, A model for collision processes in gases. I. Small amplitude processes in charged and neutral one-component systems, Phys. Rev., 94 (1954), pp. 511-525. 
[5] B. M. Boghosian, P. J. Love, And J. Yepez, Entropic lattice Boltzmann model for Burgers's equation, 362 (2004), pp. 1691-1701.

[6] A. Brezillon, G. Girault, and J. Cadou, A numerical algorithm coupling a bifurcating indicator and a direct method for the computation of Hopf bifurcation points in fluid mechanics. Comput. \& Fluids 39, 1226?1240. (2010)

[7] R. A. Brownlee, A. N. Gorban, And J. Levesley, Stabilization of the lattice Boltzmann method using the Ehrenfests' coarse-graining idea, Phys. Rev. E, 74 (2006), 037703.

[8] R. A. Brownlee, A. N. Gorban, and J. Levesley, Stability and stabilization of the lattice Boltzmann method, Phys. Rev. E, 75 (2007), 036711.

[9] R. A. Brownlee, A. N. Gorban, and J. Levesley, Nonequilibrium entropy limiters in lattice Boltzmann methods, Physica A: Statistical Mechanics and its Applications, 387 (2008), pp. $385-406$.

[10] R.A. Brownlee, J. Levesley, D. Packwood, and A.N. Gorban, Add-ons for Lattice Boltzmann Methods: Regularization, Filtering and Limiters, In: Novel Trends in LatticeBoltzmann Methods - Reactive Flow, Physicochemical Transport and Fluid-Structure Interaction, M. Ehrhardt (ed.), Progress in Computational Physics, Vol. 3, Bentham Science Publishers, 2012. arXiv:1110.0270 [physics.comp-ph].

[11] C. H. BRuneau AND M. SAAD, The $2 D$ lid-driven cavity problem revisited, Computers \& Fluids, 35 (2006), pp. $326-348$.

[12] S. Chen And G. D. Doolen, Lattice Boltzmann method for fluid flows, Annual Review of Fluid Dynamics, 30 (1998), pp. 329-364.

[13] P. J. Dellar, Non-hydrodynamic modes and a priori construction of shallow water lattice Boltzmann equations, Phys. Rev. E, 65 (2002), 036309 (12 pages).

[14] P. J. Dellar, Incompressible limits of lattice Boltzmann equations using multiple relaxation times, J. Comput. Phys., 190 (2003), pp. 351-370.

[15] P. J. Dellar, Non-hydrodynamic modes and general equations of state in lattice Boltzmann equations, Physica A: Statistical Mechanics and its Applications, 362 (2006), pp. 132 138.

[16] D. D'Humieres, Generalized lattice Boltzmann equations, in Rarefied gas dynamics, Vancouver, 1994, pp. 450-458.

[17] D. D'Humieres, I. Ginzburg, M. Krafczyk, P. Lallemand, and L.-S. Luo, Multiplerelaxation-time lattice Boltzmann models in three dimensions, Philosophical Transactions of the Royal Society of London. Series A: Mathematical, Physical and Engineering Sciences, 360 (2002), pp. 437-451.

[18] J. W. Goodrich, K. Gustafson and K. Halasi, Hopf bifurcation in the driven cavity J. Comput. Phys. 90, 219?261. (1990)

[19] A. N. Gorban, P. A. Gorban, And G. Judge, Entropy: The Markov ordering approach, arXiv:1003.1377 (2010).

[20] A. N. Gorban AND D. PACKWood, Allowed and forbidden regimes of entropy balance in lattice Boltzmann collisions, Phys. Rev. E 86, 025701(R) (2012).

[21] S. Hou, Q. Zou, S. Chen, G. Doolen, And A.C. Cogley, Simulation of cavity flow by the lattice Boltzmann method, J. Comput. Phys., 118 (1995), pp. 329-347.

[22] I. V. Karlin, A. Ferrante, and H. C. Öttinger, Perfect entropy functions of the lattice Boltzmann method, Europhys. Lett., 47 (1999), pp. 182-188.

[23] I. V. Karlin, A. N. Gorban, S. Succi, And V. Boffi, Maximum entropy principle for lattice kinetic equations, Phys. Rev. Lett., 81 (1998), pp. 6-9.

[24] I. V. Karlin, L. L. Tatarinova, A. N. Gorban, H. C. Öttinger, Irreversibility in the short memory approximation, Physica A, 327 (2003), pp. 399-424.

[25] P. Lallemand And L.-S. Luo, Theory of the lattice Boltzmann method: Dispersion, dissipation, isotropy, Galilean invariance, and stability, Phys. Rev. E, 61 (2000), pp. 6546-6562.

[26] L.-S. Luo, W. Liao, X. Chen, Y. Peng, and W. Zhang, Numerics of the lattice Boltzmann method: Effects of collision models on the lattice Boltzmann simulations, Phys. Rev. E, 83 (2011), 056710

[27] D. PackWood, Entropy balance and dispersive oscillations in lattice Boltzmann models, Phys. Rev. E, 80 (2009), 067701.

[28] D.J. Packwood, J. Levesley, and A.N. Gorban, Time step expansions and the invariant manifold approach to lattice Boltzmann models, In: Coping with Complexity: Model Reduction and Data Analysis, A.N. Gorban and D. Roose (eds.), Lecture Notes in Computational Science and Engineering, 75, Springer: Heidelberg - Dordrecht - London - New York, 2011, pp. 169-206.

[29] T. W. PAN ANd R. Glowinksi, A projection/wave-like equation method for the numerical simulation of incompressible viscous fluid flow modeled by the Navier-Stokes equations, 
Comp. Fluid Dyn. J., 9 (2000), pp. 28-42.

[30] Y. F. Peng, Y. H. Shiau, and R. R. Hwang, Transition in a 2-d lid-driven cavity flow, Computers \& Fluids, 32 (2003), pp. $337-352$.

[31] D. Ricot, S. Marie, P. SAgaut, And C. BAilly, Lattice Boltzmann method with selective viscosity filter, Journal of Computational Physics, 228 (2009), pp. 4478-4490.

[32] S. SucCI, The lattice Boltzmann equation for fluid dynamics and beyond, Numerical mathematics and scientific computation, Clarendon Press, 2001.

[33] W.-A. Yong AND L.-S. LuO, Nonexistence of $H$ theorems for the athermal lattice Boltzmann models with polynomial equilibria, Phys. Rev. E, 67 (2003), 051105.

[34] W.-A. Yong And L.-S. Luo, Nonexistence of $H$-theorem for some lattice Boltzmann models, J. Stat. Phys., 121 (2005), pp. 91-103.

[35] E. TADMOR AND W. Zhong, Entropy stable approximations of Navier-Stokes equations with no artificial numerical viscosity, J. Hyberbolic Differ. Equ., 3 (2006), pp 529-559. 